
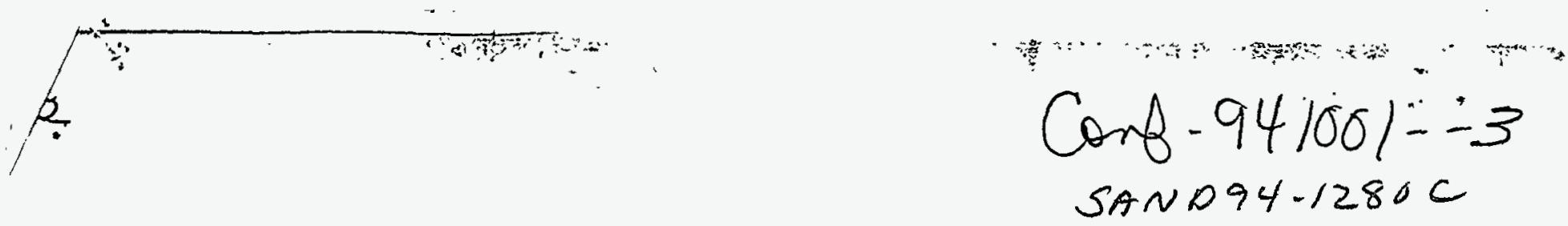

Submitted to J. Vac. Sci. Technol. A

Proceedings of the $41^{\text {st }}$ National AVS Symposium

Abstract \# : 421

Program \# : PS1-ThA8

\title{
Defect Production and Recombination During Low-Energy Ion Processing
}

B.K. Kellerman, J.A. Floro, E. Chason, D.K. Brice, S.T. Picraux, Sandia National Laboratories, Albuquerque, NM 87185

J.M. White, Science and Technology Center at the University of Texas at Austin, Austin, TX 78712.

\section{ABSTRACT}

Low-energy ion processing produces damaged, microroughened semiconductor surfaces due to the production of point defects. We present a study of point defect production and annealing on the Ge(001)-2x1 surface during low-energy inert ion bombardment as a function of ion energy, ion mass and substrate temperature. Ion-induced surface point defect production was quantified experimentally in real time using in situ Reflection High Energy Electron Diffraction. The observed surface defect yield decreased abruptly around room temperature as the substrate temperature was increased from $175 \mathrm{~K}$ to $475 \mathrm{~K}$. We have developed Monte Carlo simulations of defect diffusion to model defect recombination both in the bulk and on the surface. Bulk defect production statistics generated by our binary collision simulator, TRIMRC, were coupled with our bulk diffusion simulator to predict the number of ion-induced surface defects. A comparison between the experimental results and the simulation predictions indicated that defects produced in the bulk may represent a significant contribution to the observed surface defect yield and suggested that TRIMRC may overestimate the depth distribution of the defects. The simulations further indicated that the abrupt drop in the experimental yield with increasing substrate temperature does not arise from bulk defect recombination. Our Monte Carlo simulations of surface diffusion (applicable to any crystalline surface) support a defect annealing mechanism (at low ion fluxes) that involves surface recombination of defects generated within a single cascade. 


\section{DISCLAIMER}

This report was prepared as an account of work sponsored by an agency of the United States Government. Neither the United States Government nor any agency thereof, nor any of their employees, make any warranty, express or implied, or assumes any legal liability or responsibility for the accuracy, completeness, or usefulness of any information, apparatus, product, or process disclosed, or represents that its use would not infringe privately owned rights. Reference herein to any specific commercial product, process, or service by trade name, trademark, manufacturer, or otherwise does not necessarily constitute or imply its endorsement, recommendation, or favoring by the United States Government or any agency thereof. The views and opinions of authors expressed herein do not necessarily state or reflect those of the United States Government or any agency thereof. 


\section{DISCLAIMER}

Portions of this document may be illegible in electronic image products. Images are produced from the best available original document. 


\section{INTRODUCTION}

Semiconductor surfaces are routinely exposed to low-energy ions during cleaning, etching and deposition processes. Low-energy ions have been used on dynamic (growing) surfaces during sputter deposition and ion-assisted deposition of electronic materials[1]. Plasma-assisted chemical vapor deposition and plasma-assisted etching processes, such as reactive ion etching, involve exposing a surface to a variety of species including energetic ions.[2] In addition, the control of atomic scale surface morphology through low-energy ion bombardment has been demonstrated.[3] Bombardment of semiconductor surfaces with low-energy ions produces atomic displacements and structural defects in the bulk and on the surface. The stabilization of these defects depends on several parameters including ion energy, ion mass and substrate temperature. In addition, defect migration and defect annealing during ion bombardment play a critical role when considering damage buildup in semiconductors. The realization of atomic scale control during the processing of electronic materials with energetic ions requires a fundamental understanding of the interaction of low-energy ions with semiconductor surfaces.

In a recent study we presented measurements of surface point defect production and annealing on the $\mathrm{Ge}(001)-2 \times 1$ surface during low-energy inert ion bombardment as a function of ion energy, ion mass and substrate temperature.[4,5] The observed defect yield decreased dramatically near room temperature as the substrate temperature increased from 175 to $475 \mathrm{~K}$. The role of defect diffusion, both in the bulk and on the surface, in determining the observed surface defect yield and the temperature dependence of the yield were discussed qualitatively in these studies. In this paper we use Monte Carlo simulations of defect diffusion to model defect recombination in the bulk and on the surface. Defect production statistics generated by our binary collision simulator, TRIMRC[6], coupled with our simulation of bulk.defect diffusion, indicate that defects produced in the bulk may, under certain circumstances, represent a significant contribution to the observed surface defect yield. Our results also suggest that TRIMRC, for low ion energies/large ion masses, either underestimates the total defect yield or overestimates the depth 
distribution of the defects. We show here that our Monte Carlo simulations explain the experimentally observed temperature dependence of the surface defect yield through a defect annealing mechanism that involves surface recombination of defects. This mechanism has important implications for semiconductor processing that involves the bombardment of surfaces with energetic ions, including plasma processing and ion-assisted growth. The simulation results revealed that many defects transiently reside on the surface at elevated temperatures under lowenergy ion bombardment.

\section{SUMMARY OF EXPERIMENTAL RESULTS AND TRIMRC PREDICTIONS}

We reiterate here the primary experimental results from references 4 and 5 . In these experiments, clean, smooth $\mathrm{Ge}(001) 2 \times 1$ surfaces were bombarded in an ultra-high vacuum environment by inert gas ions in the 70-500 eV energy range. The surface defect yield (number of adatoms and surface vacancies produced per incident ion) was measured in real time by monitoring the relative intensity of the out-of-phase specular RHEED spot. For details, see ref. 4. For our purposes here, it is important to note that this technique only measures defects that reside on the surface and not in the bulk.

The defect yields due to argon, helium and xenon ion bombardment all increased with ion energy at a constant substrate temperature of $175 \mathrm{~K}$ (Figure 1). The observed defect yields increased with ion mass and ion energy. The defect yields during Xe bombardment were slightly higher than for Ar bombardment. The relative defect yield for He was a factor of 5-10 lower than the corresponding Ar/Xe defect yield.

The magnitudes of the experimentally observed defect yields are meaningless without some knowledge of the origin of the defects on the surface. In particular, are the observed defect yields representative only of defects that were created on the surface or also of bulk defects that migrated to the surface? To help answer this question, the number and partitioning of displacements produced during ion bombardment was generated by a binary collision Monte Carlo simulator. This simulator, described in more detail in ref. [6], is a modified version of the basic TRIM 
(transport of ions in matter) code[7], which uses the binary collision approximation for describing collisions between the projectile and lattice atoms. TRIMRC provides a detailed evaluation of individual cascades produced by the primary ion and its recoils including the spatial distribution of vacancies and interstitials produced on the surface and in the bulk as a function of ion mass and ion energy. We generated defect production statistics using bulk and surface displacement threshold energies of $15 \mathrm{eV}$ and $11.5 \mathrm{eV}$, respectively.[8,9]

Figure 1 shows a comparison of the TRIMRC predicted total defect yields with the experimentally observed defect yields. The TRIMRC total defect yield refers to all vacancies and interstitials produced (both on the surface and in the bulk) per incident ion. For He (Fig. 1a), the observed defect yield lies very near the TRIMRC total defect yield for ion energies below $150 \mathrm{eV}$. As the ion energy increases, the observed yield falls below the TRIMRC total defect yield. In the case of Ar and Xe bombardment (Fig. $1 \mathrm{~b}$ and 1c), the observed defect yield slightly exceeded the TRIMRC total defect yield for all ion energies.

Assuming TRIMRC predicts the cascade profiles with reasonable accuracy, these comparisons suggest that migration of bulk defects may be important. TRIMRC predicted a depth distribution of Ar/Xe-induced defects extending approximately 16 monolayers below the surface.[4] In the case of He bombardment, TRIMRC predicted defects down to approximately 60 monolayers below the surface. We used Monte Carlo simulation of defect diffusion in the bulk coupled with TRIMRC cascade profiles to relate the observed surface defect yields to the bulk displacement values.

Figure 2 shows the observed surface defect yields for $200 \mathrm{eV}$ Ar and Xe bombardment as a function of substrate temperature. At low temperatures, the defect yield is equal to the TRIMRC total defect yield, while at high temperatures, the defect yield is equal to the sputter yield (approximately 1 sputtered atom per incident ion[9]). The transition from TRIMRC total defect yield to sputter yield occurs over a $150 \mathrm{~K}$ temperature range centered around room temperature. In references 4 and 5, an analytical model was presented to describe the temperature dependence of the observed defect yield. The model argued that the fraction of bulk defects reaching the surface 
is insensitive to substrate temperature and that the decay in the observed defect yield could be explained entirely in terms of surface annealing. The model also assumed that defect annealing results from surface recombination of defects generated within a single cascade. The curve fit in Figure 2 was calculated by fixing the total defect yield and the activation energy for surface migration of adatoms and vacancies and varying the sputter yield and average interdefect separation. The average interdefect separation was optimized at approximately $5.5 \AA$, consistent with an annealing mechanism involving intracascade defect recombination. This model was tested using the Monte Carlo simulations of bulk and surface defect diffusion. The results of these simulations are given in the next two sections.

\section{SIMULATION RESULTS OF BULK DEFECT DIFFUSION}

The generation and spatial arrangement of bulk defects due to ion bombardment was predicted using the binary collision Monte Carlo simulator TRIMRC. These defects were then allowed to migrate in a bulk diffusion simulator to examine the competition between bulk recombination/clustering and escape to the surface. Defects reaching the surface were then allowed to migrate in our surface diffusion simulator to examine the competition between defect clustering (surface roughening) and defect recombination/step annihilation (surface smoothing). The results of the bulk diffusion simulations will be discussed in this section, while the results of the surface diffusion simulations will be discussed in the following section.

Our simulations of bulk diffusion involved the random walk migration of point defects through a three-dimensional simple cubic lattice. A single cascade produced from TRIMRC generated the number of each type of defect as well as their lattice coordinates. This output was

used as the initial condition for the bulk diffusion simulations. For statistical convergence, eighty different cascades were allowed to diffuse fifty times per ion mass/ion energy combination. Statistics on defect clustering, recombination and surface trapping were obtained as function of substrate temperature in the range $175 \mathrm{~K}$ to $475 \mathrm{~K}$. Point defect migration in semiconductors is 
empirically found to be thermally activated. The random walk diffusion of point defects is defined simply in terms of the jump frequency:

$$
\Gamma=v_{0} \exp (-Q / \mathrm{kT})
$$

where $\Gamma$ is the hopping frequency, $v_{0}$ is the Debye frequency, taken here as $10^{-12} \sec ^{-1}, \mathrm{Q}$ is the activation energy for migration, $\mathrm{k}$ is Boltzmann's constant, and $\mathrm{T}$ is the temperature. Activation energies for interstitial and vacancy migration were independently varied from 0.1 to $1.0 \mathrm{eV}$. The interstitials and vacancies traveled on separate cubic lattices that were offset by $\left(\frac{1}{2}, \frac{1}{2}, \frac{1}{2}\right) \mathrm{a}_{0}$, where $a_{0}$ is the lattice constant. The probability of moving a particular type of defect depended upon its activation energy for migration and the number of that type of defect relative to the total number of defects. All defects migrated isotropically with no defect-defect or surface-defect interactive forces. Defects of opposite type recombined[10] and vacancies clustered[11] when they were separated by a nearest neighbor distance. Divacancies and higher order clusters were immobile. Any defect that reached the surface during a simulation run was trapped at its position on the surface. Thus, spatial distribution profiles of defects that percolated to the surface were calculated for various ion mass/ion energy combinations. These profiles formed the initial condition for the surface diffusion simulations described in the next section.

A direct comparison between TRIMRC predictions and the observed defect yields (Fig. 1) suggested that defects produced in the bulk may represent a significant contribution to the observed surface defect yield. We will focus on answering two key questions with the bulk diffusion simulations. Can the diffusion of bulk defects in the TRIMRC cascade explain the observed surface defect yields? Is the fraction of defects reaching the surface insensitive to the substrate temperature, (an assumption of the model for the temperature dependence of the surface defect yield $[4,5])$ ?

The Monte Carlo simulation of bulk diffusion generated statistics on defect clustering, recombination and surface trapping as a function of substrate temperature and relative activation energies for migration. Figure 3 shows the results of defect recombination/clustering and surface trapping for each ion at $175 \mathrm{~K}$. The fraction of point defects that migrated further into the bulk (not 
shown) was negligible for $\mathrm{Ar} / \mathrm{Xe}$ bombardment and approximately $10 \%$ for $\mathrm{He}$ bombardment. This is consistent with TRIMRC predictions of the depth distribution of ion-induced defects being approximately four times deeper during He bombardment compared to Ar/Xe bombardment. The fraction of defect recombination/clustering and surface trapping are plotted as a function of cascade depth and ion energy. The cascade depth is defined as the depth at which the cumulative total defect fraction reached $95 \%$ of the total defects. For low ion energies, more than half of the bulk defects recombined or clustered in the bulk and never reached the surface. As the incident ion energy increased, the fraction of defects that escaped to the surface decreased, while the fraction of defects that recombined/clustered increased. Within the energy range examined here, higher ion energies produced denser cascades, enhancing recombination. In fact, most of the recombination/clustering events occurred in the initial stages of diffusion, amongst defects that were initially close neighbors after cascade quenching. Additionally, higher energy ions produced cascades deeper in the solid, further reducing the probability of defects escaping to the surface.

The fraction of defects that reached the surface were converted into surface defect yields for a direct comparison between the predicted number of ion-induced surface defects with the experimental surface defect yields. Figure 1 compares the predicted surface defect yields with the observed surface defect yields. In the case of He bombardment (Fig. 1a), the random walk diffusion of bulk defects coupled with TRIMRC cascade profiles adequately predicted the surface defect yield for ion energies above approximately $300 \mathrm{eV}$. The binary collision approximation is assumed to be valid for light ions down to $100 \mathrm{eV}$.[7] However, the disagreement between the predicted and experimental surface defects yields suggests that the binary collision approximation may not hold for He bombardment at energies below $300 \mathrm{eV}$. The fraction of defects reaching the surface was insensitive to the relative activation energies. This insensitivity enabled an upper limit on the activation energies for migration of point defects in germanium to be predicted. Varying the activation energies until the predicted surface defect yield during $\mathrm{He}$ bombardment deviated significantly from the observed defect yield placed an upper limit on the activation energies of migration for both interstitials and vacancies at $0.32 \pm 0.03 \mathrm{eV}$. This value is consistent with 
radiation damage experiments that placed an upper limit on the activation energies for diffusion of both single vacancies and single interstitials at $0.30 \mathrm{eV} .[12]$

In the case of $\mathrm{Ar} / \mathrm{Xe}$ bombardment (Figs. $1 \mathrm{~b}$ and $1 \mathrm{c}$ ), the predicted surface defect yield falls well below the total defect yield over the entire energy range. The relatively low fraction of defects escaping to the surface in the TRIMRC cascades (Figs. 3b and 3c) suggests that TRIMRC underestimates the total defect yield or overestimates the depth distribution of defects. The shortcomings of the predicted surface defect yields arise from the binary collision approximation used in TRIMRC. The binary collision approximation becomes increasingly inaccurate as the ion mass increases and the ion energy decreases. In the low energy/high mass regime, the creation of a cascade is a many-body process with a high local energy density, and calculations using the binary collision approximation are likely to underestimate the total defect yield and/or overestimate the depth distribution. Molecular dynamics simulations of $200 \mathrm{eV}$ Xe bombardment of silicon at $60^{\circ}$ off-normal angle of incidence produced much shallower cascades, with the ion-induced defects confined within the first few monolayers[13]. Defect production statistics were not obtained in these simulations. However, molecular dynamics simulations of self-bombardment of silicon in the $100 \mathrm{eV}$ range indicated that predictions of MARLOWE[14] (another Monte Carlo simulation that uses the binary collision approximation to generate defect production statistics) underestimated the total defect yield by about 50\%.[15] Thus, the cascade depth/density is of critical importance to determining the observed surface defect yield.[16]

References 4 and 5 argued qualitatively that the fraction of defects reaching the surface is insensitive to the substrate temperature, i.e. the observed temperature dependence was not due to bulk recombination processes. Bulk recombination has been used to explain a similar temperature dependent damage yield observed with higher energy ion bombardment of germanium.[17] However, our bulk diffusion simulator indicated that for low ion fluxes the fraction of defects that reached the surface was essentially independent of the substrate temperature, changing by less than $5 \%$. Increasing the substrate temperature will increase the bulk diffusivity of the defects. However, the higher diffusivity of vacancies and interstitials increases both the rate at which 
defects recombine and the rate at which defects reach the surface. Thus, although the kinetic rates do vary strongly with temperature, the fraction of defects that reach the surface remains relatively insensitive to the substrate temperature. Inasmuch as our experiments sampled only those defects residing on the surface, and because the fraction of bulk defects that reach the surface is insensitive to the substrate temperature, defect annealing in our experiments must be taking place on the surface.

\section{SIMULATION RESULTS OF SURFACE DEFECT DIFFUSION}

The temperature dependence of the surface defect yield (Fig. 2) was modeled in references 4 and 5 as resulting from surface recombination of point defects all generated within the same cascade. We used Monte Carlo simulations of surface defect diffusion to evaluate an annealing mechanism that involves surface recombination of defects to further explore the observed temperature dependent behavior.

Our modeling of surface diffusion of adatoms and monovacancies involved the random walk of point defects on a square surface mesh having the same lattice constant as the bulk.[18] For each ion impingement event, an $(x, y)$ coordinate pair was randomly chosen on the surface. The number of defects that corresponded to the experimentally observed total defect yield were placed around this $(x, y)$ location. The placement of the defects was weighted by the distribution profiles generated from the bulk diffusion simulations. The defects then were allowed to migrate isotropically on the surface until they clustered, recombined or annihilated at a step edge. Activation energies for diffusion of adatoms and vacancies were independently varied between 0.7 $-1.0 \mathrm{eV}$. A value of $0.8 \mathrm{eV}$ has previously been measured for the surface diffusion of vacancies and adatoms on $\mathrm{Ge}(001)$.[19] Note that the activation energies for the surface diffusion of vacancies/adatoms is larger than the respective activation energies for bulk diffusion.[12] The activation energies for jumping across steps, jumping to and from an island, edge diffusion, and detachment of one-, two- and three-coordinated atoms could be varied independently but were typically held at the same values of $0.0 \mathrm{eV}, 0.8 \mathrm{eV}, 1.0 \mathrm{eV}, 1.0 \mathrm{eV}, 1.2 \mathrm{eV}, 1.4 \mathrm{eV}$, respectively. 
Out-of-phase specular RHEED intensities were calculated as the surface evolved during each simulation run to determine the observed defect yield.

Our surface diffusion simulator revealed that both intracascade and intercascade recombination are responsible for the decay in the observed defect yield from total defect yield to sputter yield. As Figure 2 shows, the simulations predicted a transition region from total defect yield to sputter yield centered around room temperature. The simulations indicated that the primary annealing mechanism is the recombination of defects within a single cascade, however, intercascade recombination and step-edge annihilation become more important as the temperature increases. Thus, a second, larger length scale for defect recombination and annihilation becomes more important as the defects become sufficiently mobile.

Figures 4 and 5 schematically illustrate the defect production and surface defect annealing mechanism we believe is occurring during low-energy ion bombardment and low ion fluxes. An ion incident upon the germanium surface produces a cascade of point defects, possibly extending into the bulk (Fig. 4a). Point defects are very mobile in germanium even at temperatures below $200 \mathrm{~K}$ and any bulk migration of point defects to the surface occurs quickly (Fig. 4b). Bulk defects initially in close proximity to one another will either recombine or cluster and never reach the surface. Defects that have reached the surface at substrate temperatures below approximately $250 \mathrm{~K}$ are immobile (Fig. 5a) and hence contribute to the observed total defect yield. For substrate temperatures above approximately $250 \mathrm{~K}$, the point defects become mobile on the surface. Initially, the defects are able to hop only a few steps, and if they recombine, it will most likely be with an opposite-type defect produced within the same cascade (Fig. 5b). Thus, the observed defect yield is slightly less than the total defect yield. As the substrate temperature continues to increase, the defects become sufficiently mobile to travel long distances $(\geq 100 \AA)$. If the defect does not immediately recombine or cluster with a defect produced within the same cascade, it will migrate away from the cascade and recombine/cluster with a defect from another cascade or annihilate at a step edge (Fig. 5c). As a result, all that can remain after complete recombination of 
vacancies/adatoms are the uncompensated sputter vacancies, and hence the observed surface defect yield is the sputter yield.

This mechanism has important implications for processing of semiconductor surfaces with low-energy ions, especially beam-assisted epitaxial film growth. Although the observed defect yield may be on the order of one defect/ion (i.e., the sputter yield) for substrate temperatures at typical growth temperatures, an order of magnitude more defects transiently reside on the surface prior to recombining or annihilating and may help destabilize small clusters. These short-lived defects play a significant role in modifying the growth process and will likely affect the resulting surface morphology of the film.

\section{CONCLUSIONS}

We used Monte Carlo simulations of defect diffusion to model defect recombination in the bulk and on the surface to lend insight into experimental results. The observed surface defect yields for $\mathrm{Ar} / \mathrm{Xe}$ bombardment nearly equaled the TRIMRC total defect yields suggesting bulk diffusion of defects may be important. However, predictions of the surface defect yield using a combination of the TRIMRC predicted total defect yield, coupled with a simulation of bulk diffusion of point defects, showed that a large fraction of bulk defects recombine/cluster and never reach the surface, inconsistent with our experimental results. This suggests that TRIMRC underestimates the total defect yield or overestimates the depth distribution. The observed surface defect yields for He bombardment were predicted reasonably well for He ion energies above approximately $300 \mathrm{eV}$. Bulk defects may be contributing to the observed defect yield in all cases, indicating a high mobility of point defects in germanium $(\leq 0.35 \mathrm{eV})$.

The experimental defect yield was found to decrease from the total defect yield to the sputter yield as the substrate temperature increased from $175 \mathrm{~K}$ to $475 \mathrm{~K}$. The model for the temperature dependence of the defect yield in references 4 and 5 assumed that the decay in the observed surface defect yield could be explained entirely in terms of intracascade surface recombination. Our Monte Carlo simulations of bulk defect diffusion indicate that the fraction of 
bulk defects that reach the surface is relatively independent of the substrate temperature. Thus, the observed drop in the surface defect yield with temperature must result from surface annealing. The simulations also showed that intracascade surface recombination becomes important at sufficiently high temperature. At substrate temperatures below approximately $250 \mathrm{~K}$, surface defects are immobile and contribute to the total defect yield. At intermediate temperatures, recombination of defects will primarily involve defects generated within a single cascade (i.e., intracascade recombination). As the temperature continues to increase, defects become sufficiently mobile to allow intercascade recombination and annihilation at step edges. Although the observed defect yield is only about one defect/ion at typical growth temperatures, an order of magnitude more defects transiently reside on the surface prior to recombining or annihilating. These defects can significantly affect growth morphology when concurrent growth and bombardment are performed. This result has important implications for semiconductor processing that involves the bombardment of surfaces with energetic ions, including plasma processing and ion-assisted growth.

\section{ACKNOWLEDGMENTS}

We would like to acknowledge Brian S. Swartzentruber for many helpful discussions. This work was performed at Sandia National Laboratories and supported by the United States Department of Energy under Contract DE-AC04-94AL85000. JMW acknowledges the support of the National Science Foundation under grant CHE 8920120. 


\section{REFERENCES}

email:\{bkkelle,jafloro,ehchas,picraux\}@somnet.sandia.gov

1. E. Chason, P. Bedrossian, K.M. Horn, J.Y. Tsao, and S.T. Picraux, Appl. Phys. Lett. 57, 1793 (1990), and C.J. Tsai, P. Rozenak, T. Vreeland, and H.A. Atwater, J. Cryst. Growth 111, 931 (1991).

2. S. Veprek, Thin Solid Films, 130, 135 (1985), and G.A. Vawter and J.R. Wendt, Appl. Phys. Lett. 58, 289 (1991).

3. E. Chason, T.M. Mayer, B.K. Kellerman, D.N. McIlroy, and A.J. Howard, Phys. Rev. Lett. 72, 3040 (1994), and E. Chason, T.M. Mayer, and A.J. Howard, Màt. Res. Soc. Symp. Proc. 317, 91 (1994).

4. J.A. Floro, B.K. Kellerman, E. Chason, S.T. Picraux, D.K. Brice, and K.M. Horn, J. Appl. Phys. (submitted).

5. J.A. Floro, B.K. Kellerman, E. Chason, S.T. Picraux, D.K. Brice, and K.M. Horn, Mat. Res. Soc. Symp. Proc. 316, 993 (1994).

6. D.K. Brice, J.Y. Tsao, and S.T. Picraux, Nucl. Instr. \& Meth. B44, 68 (1989).

7. J.P. Biersack and L.G. Haggmark, Nucl. Instr. \& Meth. 174, 257 (1980).

8. Y. Chen and J.M. MacKay, Phil. Mag. 19, 357 (1969).

9. J.L. Vossen and J.J. Cuomo, in Thin Film Processes, John L. Vossen and Werner Kern, eds. (Academic Press, New York, 1978), p. 17.

10. K.M. Klein, Ph.D. dissertation, Department of Electrical Engineering, University of Texas at Austin, 1991.

11. M. Bertolotti, Radiation Effects in Semiconductors, edited by F.L. Vook (Plenum Press, New York, 1968), p. 311.

12. A. Seeger and K.P. Chik, Phys. Stat. Sol. 29, 455 (1968).

13. K.-H. Heinig, D. Stock, H. Boettger, V.A. Zinovyev, A.V. Dvurechenskii, and L.N. Aleksandrov, Mat. Res. Soc. Symp. Proc. 316, 1035 (1994).

14. M.T. Robinson and I.M. Torrens, Phys. Rev. B 9, 5008 (1974). 
15. George Gilmer, private communication.

16. Alternatively, TRIMRC may not underestimate the total defect yield but diffusion enhancement mechanisms may account for the large number of $\mathrm{Ar} / \mathrm{Xe}$ ion-induced defects reaching the surface. We have previously addressed the role of several potential enhancement mechanisms including cascade-assisted self diffusion, electrostatic image forces and elastic image forces.[4] We believe none of these enhancement mechanisms contribute significantly, if at all.

17. T.E. Haynes and D.W. Holland, Appl. Phys. Lett. 59, 452 (1991).

18. E. Chason and B.W. Dodson, J. Vac. Sci. Technol. A 9, 1545 (1991).

19. E. Chason, J.Y. Tsao, K.M. Horn, S.T. Picraux, and H.A. Atwater, J. Vac. Sci. Technol. A 8, 2507 (1990). 


\section{FIGURE CAPTIONS}

Figure 1. Comparison of the predicted surface defect yields and TRIMRC predicted total defect yields with the experimentally observed surface defect yields: (a) He bombardment, (b) Ar bombardment, (c) Xe bombardment of $\mathrm{Ge}(001)$.

Figure 2. The temperature dependence of the observed and simulated defect yields for $200 \mathrm{eV} \mathrm{Ar}$ and Xe ion bombardment. The solid line is a fit of a model for the temperature dependence described in detail in references 4 and 5 .

Figure 3. Fraction of defects that either reached the surface or recombined/clustered during bulk defect migration of ion-induced defects: (a) He bombardment, (b) Ar bombardment, (c) Xe bombardment of $\mathrm{Ge}(001)$.

Figure 4. Schematic of cascade production during low-energy ion bombardment: (a) ion impact creating the initial cascade of point defects, possibly extending into the bulk, (b) subsequent migration of bulk defects resulting in increased density of surface defects, vacancy clustering, bulk recombination and defects migrating further into the bulk.

Figure 5. Schematic of the evolution of ion-induced cascades on the surface as a function of substrate temperature: (a) low temperatures $\left(T_{S} \leq 250 \mathrm{~K}\right)$, surface defects are immobile and the total defect yield is observed, (b) intermediate temperatures ( $\left.250 \mathrm{~K}<\mathrm{T}_{\mathrm{S}} \leq 290 \mathrm{~K}\right)$, onset of surface defect mobility, intracascade recombination occurs and the observed yield is reduced, (c) high temperatures $\left(T_{S}>290 \mathrm{~K}\right.$ ), surface defects are mobile, intra/intercascade recombination occurs along with annihilation at step edges, and the sputter yield is observed. 

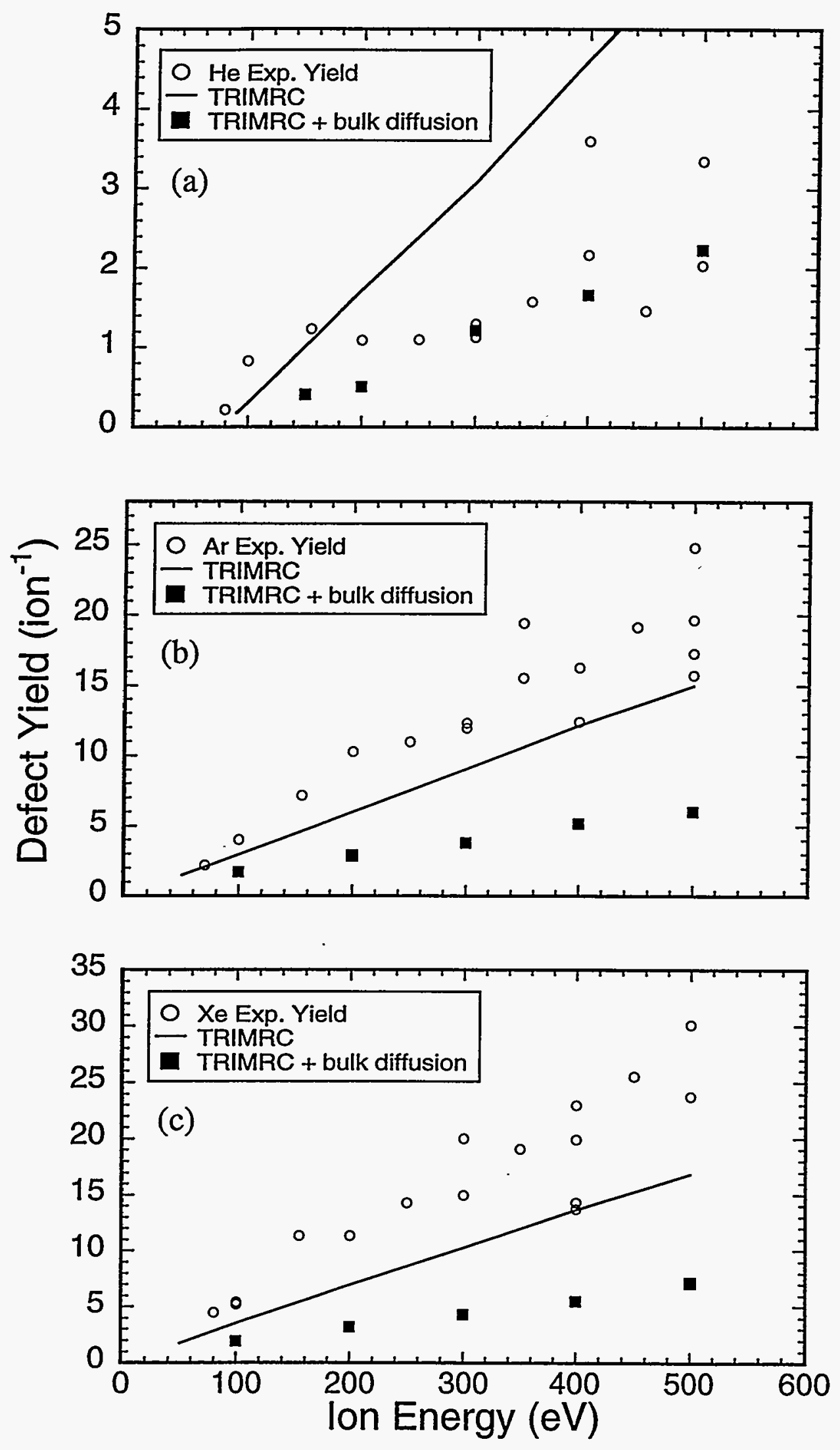


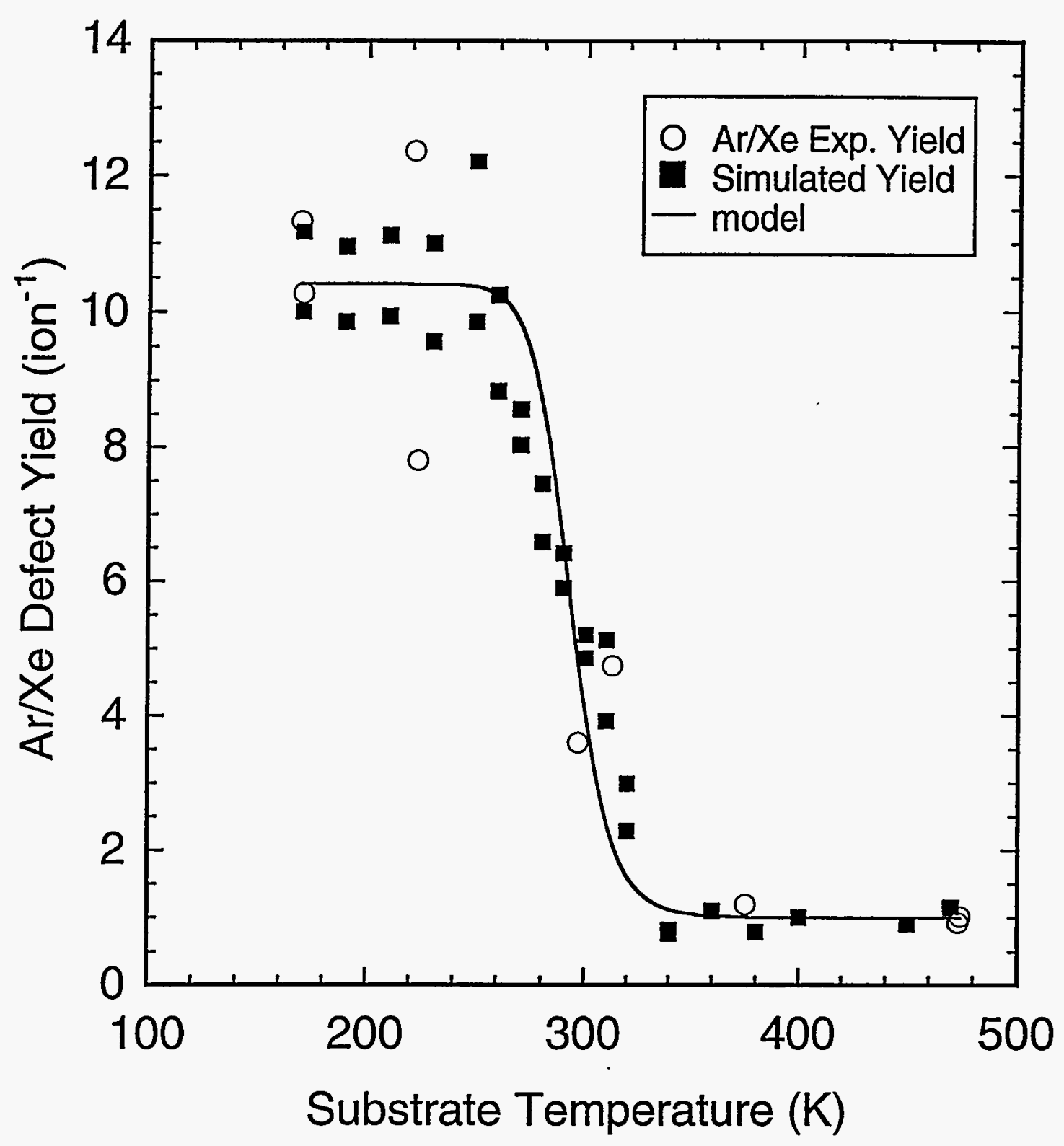



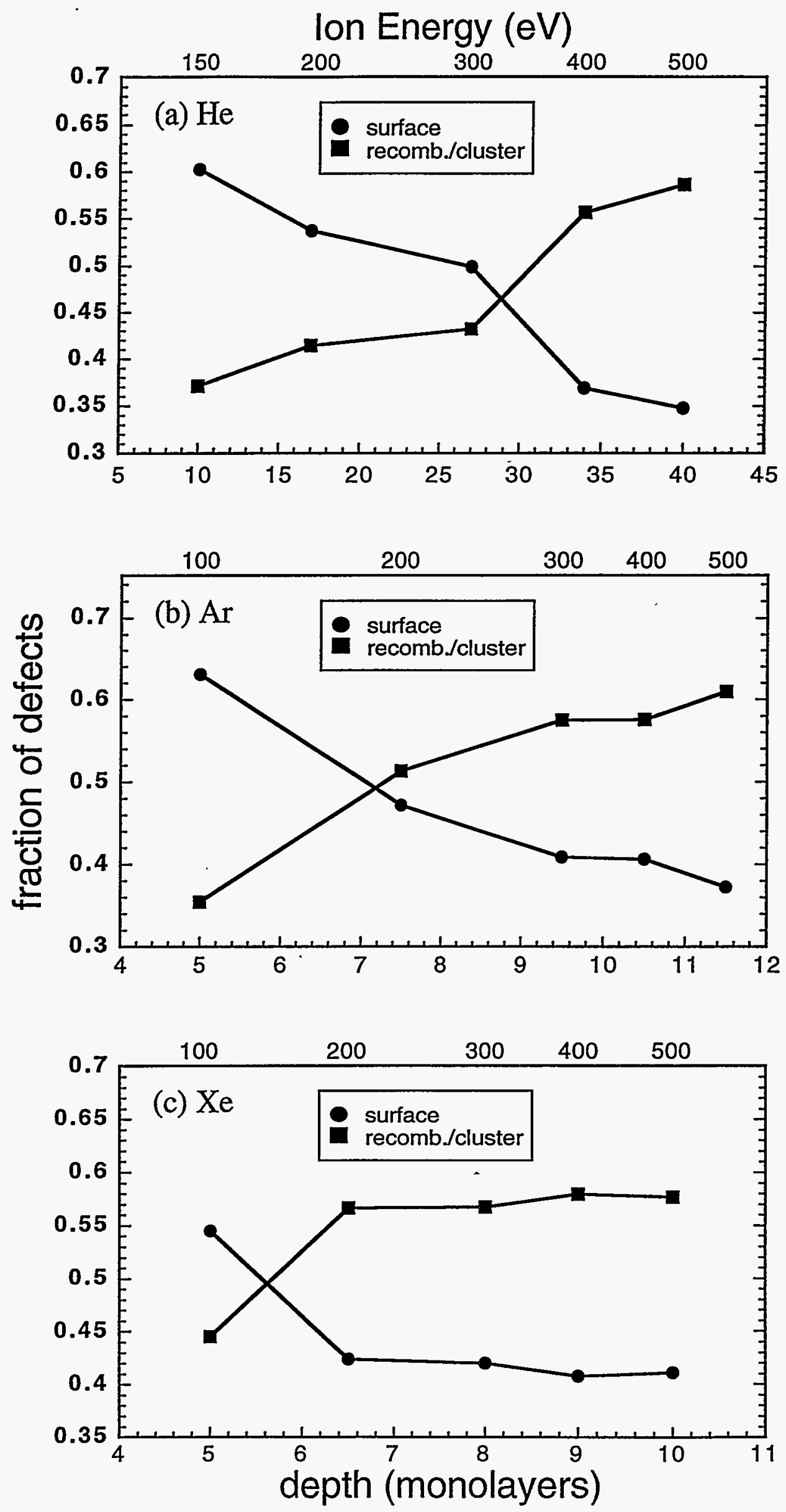


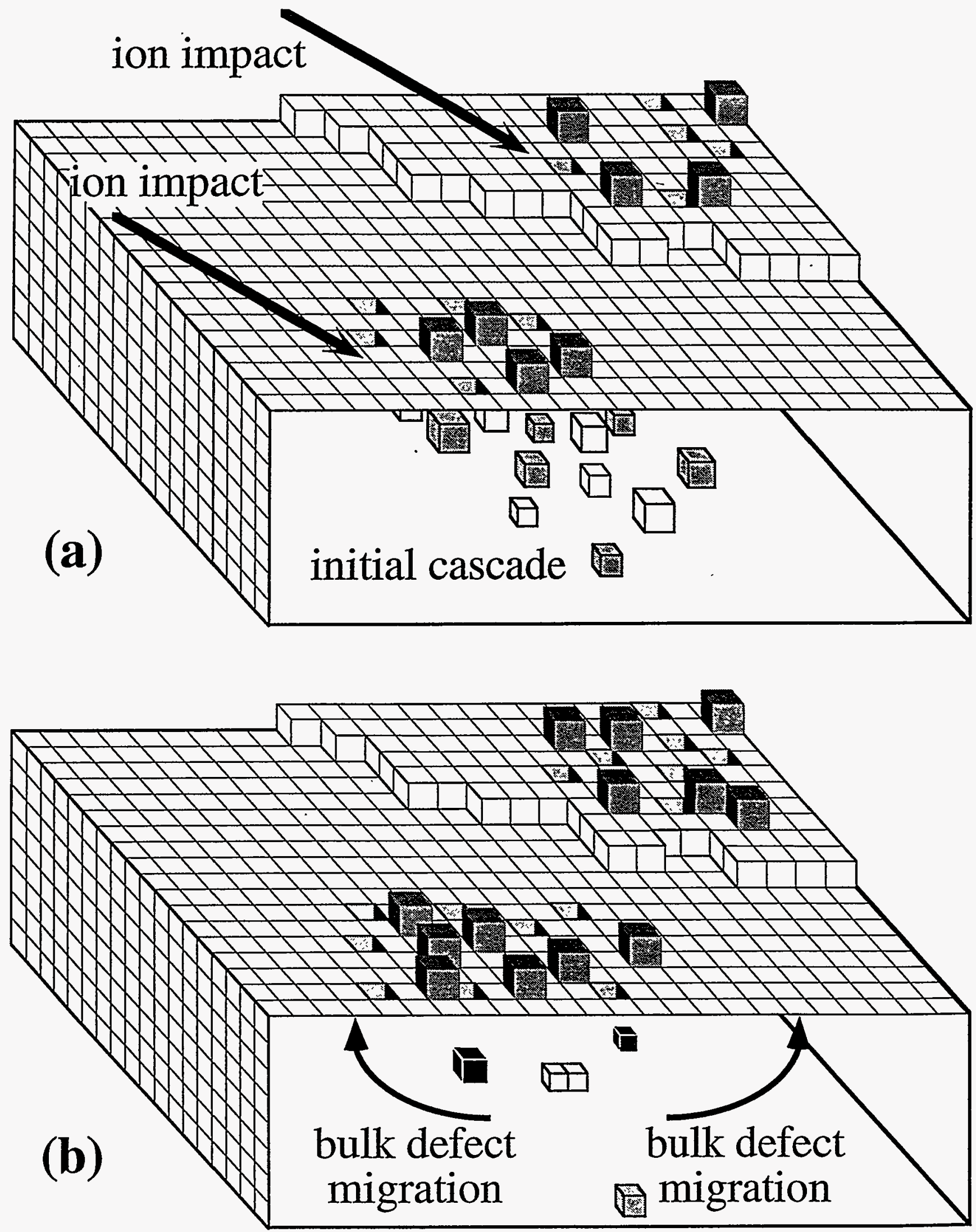

adatom

interstitial

E bulk recombination

surface vacancy $\boxminus$ bulk vacancy $\boxplus$ divacancy 

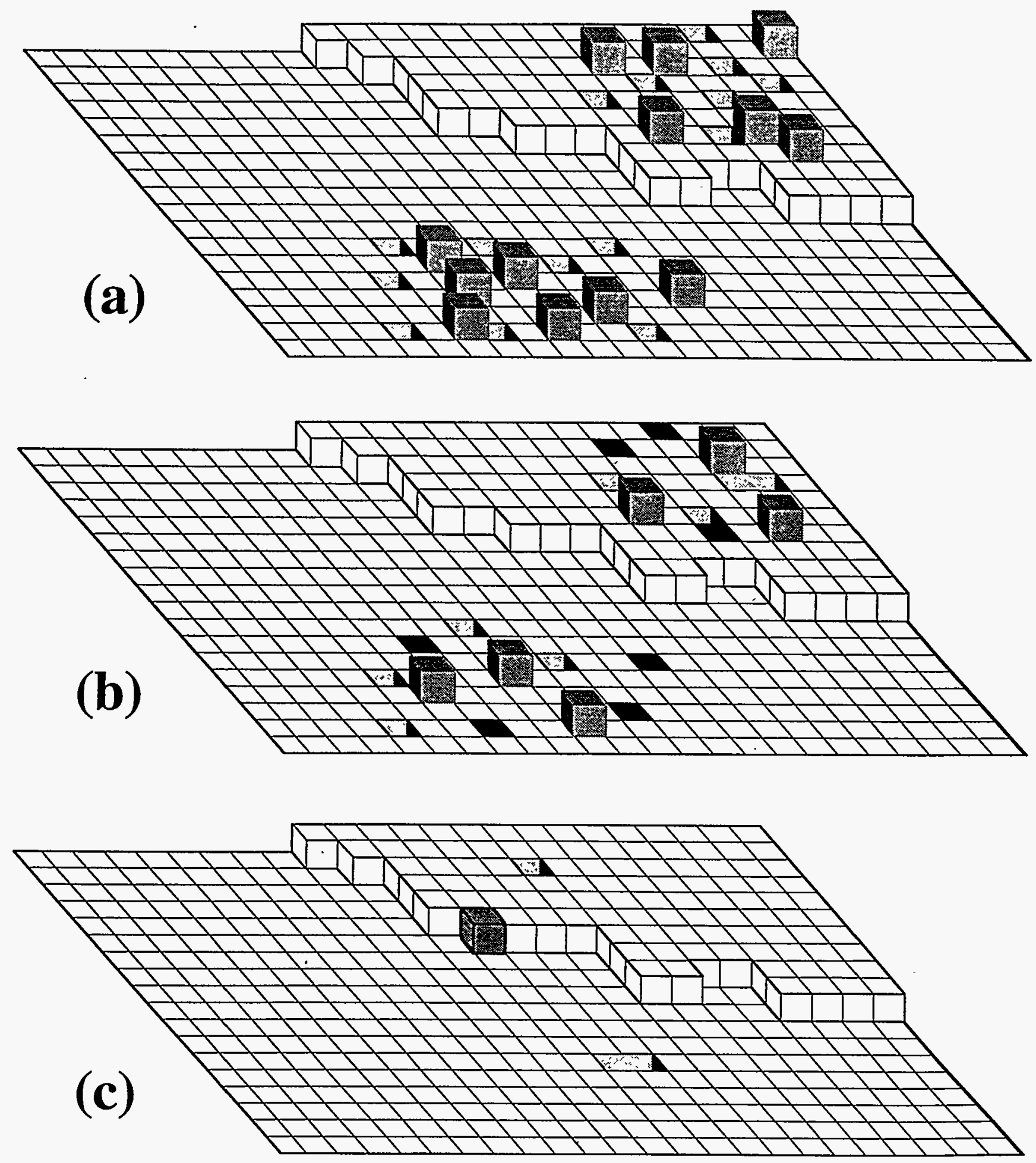

adatom

- surface recombination

- surface vacancy annihilation at step edge 